\title{
Øystein vs Archimedes: A Note on Linnebo's Infinite Balance
}

DANIEL HOEK, VIRGINIA TECH, JUNE 2021, DANIELHOEK.COM. PLEASE CITE PUBLISHED VERSION. ${ }^{1}$

\begin{abstract}
Using Riemann's Rearrangement Theorem, Øystein Linnebo (2020) argues that, if it were possible to apply an infinite positive weight and an infinite negative weight to a working scale, the resulting net weight could end up being any real number, depending on the procedure by which these weights are applied. Appealing to the First Postulate of Archimedes' treatise on balance, I argue instead that the scale would always read $0 \mathrm{~kg}$. Along the way, we stop to consider an infinitely jittery flea, an infinitely protracted border conflict, and an infinitely electric glass rod.
\end{abstract}

Imagine a perfectly symmetric balance scale in a uniform gravitational field, with a qualitatively identical object placed in each pan of the scale, at equal distances from the centre. Would this balance lean to the left, or would it lean to the right? Neither, you say: the two objects weigh the same, so there should be equilibrium. The situation is totally symmetric, so there is nothing to incline the balance in one direction over the other. Would it change your answer if I told you that the two identical weights are both infinitely heavy, and that the parts of the balance are made of an unbreakable, unbendable material? Although we are now in the realm of science fiction, it seems like it should not make a difference: the situation remains perfectly symmetric, after all.

The first postulate of Archimedes' On the Equilibrium of Planes has it that on a balance, "equal weights at equal distances are in equilibrium" (Heath 1897, p. 189). There is no restriction to finite weights -nor should there be, if the intuition just articulated is sound. Generalising slightly from this postulate, so that it applies to spring as well as balance scales, we get the following principle:

Balance Principle. When equal weights and counterweights are applied to a scale, the scale is in the same state as when no weights are applied to it.

In the case of a spring scale, the counterweights can be understood as upward "pulls" or negative weights on the scale, like helium balloons. The aim of this note is to defend the Balance Principle, in its general form, from an elegant thought experiment Øystein Linnebo proposed in this journal.

Imagine an infinite stock of brass weights of $1 \mathrm{~kg}, 1 / 3 \mathrm{~kg}, 1 / 5 \mathrm{~kg}$, and so on; and an infinite stock of counterweights of $1 / 2 \mathrm{~kg}, 1 / 4 \mathrm{~kg}, 1 / 6 \mathrm{~kg}$, and so on. At $1 \mathrm{pm}$, we begin alternately placing a weight on the left of our indestructible balance, and a counterweight on the right. We start with the biggest weights and work our way down. Or, as in Linnebo's version of the story, we alternately apply a weight and attach a balloon to a spring scale. We speed up the steps as we go, so that at 2pm exactly, all steps have been performed. Which way will the balance lean after $2 \mathrm{pm}$ ? And what will the spring scale indicate?

Since, at $2 \mathrm{pm}$, we have amassed both an infinite weight and an infinite counterweight, ${ }^{2}$ the Balance Principle predicts that the balance reaches equilibrium, and that the spring scale will point to $0 \mathrm{~kg}$. Linnebo disagrees with that prediction. He points out that, as weights and counterweights are added to the scale, the net sum of weights in favour of counterweights converges to a positive value:

$$
1-\frac{1}{2}+\frac{1}{3}-\frac{1}{4}+\ldots=\sum_{n=0}^{\infty} \frac{(-1)^{n}}{n+1}=\ln (2) \approx 0.69
$$

${ }^{1}$ Erkenntnis, online first, 2021: 1-6. Thanks to Chris Scambler and an anonymous reviewer for comments on an earlier draft, to Dane Stocks for research assistance, and to Øystein Linnebo for his delightful paradox. 
Linnebo concludes that, at $2 \mathrm{pm}$, the weights outweigh the counterweights by exactly $\ln (2) \mathrm{kg}$. So the balance scale will tilt left, and the spring scale will indicate $0.69 \mathrm{~kg}$ rather than $0 \mathrm{~kg}$ - contrary to the Balance Principle. As Linnebo observes, this way of calculating the net weight has bizarre consequences. For by Riemann's Rearrangement theorem, the limit of this conditionally convergent series depends on the order of the terms - in fact, a suitable shuffle can yield any real value whatsoever. So if Linnebo is right, the net weight pressing on the scale is not a function of what is presently on the scale, in cases like these. Instead, it is determined by the order in which the various items were added to the scale. Thus, writes Linnebo, the possibility of an actual infinity of weights and counterweights appears to imply "a dramatic departure from physics as we know it."

It seems to me, however, that these conclusions are the product of a kind of mathematical illusion. It is true that the total weight and counterweight on the balance differ at all times prior to $2 \mathrm{pm}$, and also that this weight difference converges to a positive value. But it does not follow that the difference attains its limit value, or indeed persists at all (cf. Benacerraf 1962). After all, the total weight and counterweight end up equal — such are the peculiarities of infinite arithmetic. To make this visual, imagine that the incremental weights we place on the balance are brass cylinders of uniform density and diameter, with varying heights. As we stack these cylinders neatly on top of one another, the stack on each pan grows taller and taller, with its height in inches matching its weight in kilograms. At all stages prior to $2 \mathrm{pm}$, the left tower is a bit ahead of the one on the right: it is a bit over half an inch taller and a bit over half a kilo heavier. Nonetheless, at $2 \mathrm{pm}$ two infinitely tall brass cylinders will have been completed, which do not differ in weight any more than they differ in height. If we were to fuse the segments at each stage, the two brass towers could even be qualitatively identical in all respects.

Linnebo assumes that quantities like weight must obey something like the following constraint:

Continuity Principle. If a certain natural quantity converges to a particular limit value $l$ over the course of a certain time interval $\left[t_{0}, t_{1}\right)$, and nothing further happens to affect its value at $t_{1}$, then the quantity in question attains the limit value $l$ at $t_{1}$.

This assumption is natural enough - as far as I know, there are no counterexamples to it in nature. But then, there are no supertasks in nature either — no infinite-step procedures that are completed in finite time. I submit that, in the presence of supertasks, the Continuity Principle fails for independent reasons. I just gave a counterexample: the height difference between the two brass cylinders vanishes instantaneously at $2 \mathrm{pm}$. For another counterexample, consider what happens when we empty an infinite sack of numbered apples, one by one. When the task is completed, there is nothing left in the sack. But at all times prior to completion, the number of apples remaining is infinite. Thus the number of apples in the sack drops instantaneously from infinity to zero. ${ }^{3}$

Could there be a restricted version of the Continuity Principle that avoids such counterexamples, while still sustaining Linnebo's reasoning? I think not: the principle has absurd consequences even when it is applied only to the spring scale of Linnebo's original scenario. To see this, consider what

2 By an "infinite weight," I mean a countably infinite weight. I will not consider the possibility of even greater, uncountable weights (if, indeed, it is a possibility). Likewise for infinite heights and other infinite quantities.

${ }^{3}$ A similar discontinuity occurs in the Ross-Littlewood paradox (Allis and Koetsier 1991). Begin with an empty urn. At the $n$th step of the supertask, add ten balls numbered (10n-9) up to $10 n$, and remove ball number $n$. Though the total number of balls in the urn approaches infinity, no ball survives until the end. 
would happen on this picture when, having alternately applied all the weights and counterweights to the scale in the manner described, we now remove a weight from the scale - for instance the $1 / 5 \mathrm{~kg}$ weight. On Linnebo's picture, this weight makes an essential contribution to the reading on the scale after 2pm: without it, the net weight in kilos would have converged to $\ln (2)-1 / 5$. Since this weight continues to depress the scale, the net weight on the scale should go down by $1 / 5 \mathrm{~kg}$ as soon as it is removed. 4 The same is true, mutatis mutandis, of the other component weights and counterweights.

So far, so good. But now imagine that we sequentially removed all the weights and counterweights from the scale between $2 \mathrm{pm}$ and $3 \mathrm{pm}$. Moreover, suppose we proceed in a slightly different order, releasing two counterweights after each positive weight, instead of only one. Without discontinuity, the scale pointer will then indicate the following value after $3 \mathrm{pm}: 5$

$$
\ln (2)-1+\frac{1}{2}+\frac{1}{4}-\frac{1}{3}+\frac{1}{6}+\frac{1}{8}-\frac{1}{5}+\ldots=\frac{\ln (2)}{2} \approx 0.35
$$

But that would be absurd! At 3pm all weights and counterweights have been removed, and there is nothing left on the scale. Clearly the net weight is then $0 \mathrm{~kg}$, and not $0.35 \mathrm{~kg}$. So not even a highly restricted version of the Continuity Principle can hold true in this context.

Linnebo is right that Riemann's Rearrangement theorem can teach us a lesson about supertasks. But the lesson is not that such tasks are metaphysically impossible, or that they are inconsistent with such fundamental physical precepts as the Balance Principle. Rather, it is that convergence to a limit is at best a highly defeasible heuristic for predicting the result of a supertask, because the Continuity Principle cannot be trusted in this context.

It is natural to ask how this lesson should affect our assessment of other "conditionally convergent" supertasks, involving other quantities. For example, Linnebo describes the case of a flea on a real number line. The flea starts its infinite journey at 0 . Then it hops one inch to the right to land on 1 , then $1 / 2$ inch back to the left, $1 / 3$ inch to the right and so on. Where does Linnebo's flea end up? Alternatively, imagine an infinite territorial negotiation. The Kingdom of Annexia acquires 1 square mile from its neighbour in return for a different, $1 / 2 \mathrm{mi}^{2}$ patch, then acquires $1 / 3 \mathrm{mi}^{2}$ in exchange for $1 / 4$ $\mathrm{mi}^{2}$, etcetera. How much territory does Annexia gain? Or suppose we used a silk cloth to rub off some electrons from a glass rod, until the rod achieves a positive charge of 1 coulomb. Next we let $-1 / 2$ coulomb of electrons flow back into the rod, then rub off another $1 / 3$ coulomb, and so on. What is the charge in the rod when all steps have been completed?

${ }^{4}$ By contrast, the Balance Principle predicts that no component weight or counterweight individually makes an essential contribution to the final net weight, since each one is only finite. So on the picture I advocate, the spring scale would stay balanced at $0 \mathrm{~kg}$ if any individual weight or balloon were removed.

5 To see that the sum of the removed weights converges to $1 / 2 \ln (2)$, note that:

$\begin{array}{r}\ln (2)=1+0-\frac{1}{2}+\frac{1}{3}+0-\frac{1}{4}+\frac{1}{5}+\ldots \\ -\frac{1}{2} \cdot \ln (2)=0-\frac{1}{2}+\frac{1}{4}+0-\frac{1}{6}+\frac{1}{8}+0-\ldots \\ \hline \frac{1}{2} \cdot \ln (2)=1-\frac{1}{2}-\frac{1}{4}+\frac{1}{3}-\frac{1}{6}-\frac{1}{8}+\frac{1}{5}-\ldots\end{array}$

The top row is the familiar identity $\ln (2)=\sum_{n=0}^{\infty} \frac{(-1)^{n}}{n+1}$ with zeroes interspersed at regular intervals. The middle row is the result of multiplying all terms of that identity by $-1 / 2$, and interspersing zeroes. The final row is obtained by adding the first two together. 
One upshot of our discussion is that we cannot uncritically apply the Continuity Principle in these other cases either. Might a generalised Balance Principle supply the answers instead? I suspect not. These supertasks are in fact much more diverse than meets the eye, and in my estimation the answer is different in each case. For instance, the outcome of Annexia's land swaps turns on a legal complication: who owns the areas that are swapped back and forth ad infinitum, and consequently have no final claimant? What we can say about Linnebo's flea depends on the generality of the locality principle in physics. And the analysis of the glass rod example raises a metaphysical issue about the individuation of particular electrons as they enter and exit the rod. ${ }^{6}$

Thus each supertask raises its own, subject-specific set of questions. No single, unified principle could possibly settle all the varied physical, philosophical and even legal issues that can arise. Hence I will not propose a generalised, catch-all replacement for the Continuity Principle. Instead, I incline to a kind of particularism about supertasks. My aim in this paper was to defend the Balance Principle as it applies to Linnebo's infinite scale and balance. As for other supertasks, I am afraid there just is no general answer. Supertasks resist generalisation, and there is no shortcut to carefully considering each one on its own terms - that is the bad news. But the good news is that we need not abandon our most basic physical precepts and intuitions in forming those predictions. In particular, Archimedes' balance postulate withstands Linnebo's ingenious challenge.

\section{References}

Allis, Victor and Teunis Koetsier, 1991, "On Some Paradoxes of the Infinite." British Journal for the Philosophy of Science 42 (2):187-194.

Benacerraf, Paul, $\quad$ 1962, “Tasks, Super-Tasks and the Modern Eleatics." Journal of Philosophy, 59(24): 765-784. Heath, T.L., $\quad$ 1897, The Works of Archimedes. Dover Publications.

Huggett, Nick, $\quad$ 1999, "Atomic Metaphysics." Journal of Philosophy 96(1): 5-24.

Linnebo, Øystein, 2020, “Riemann's Scale: A Puzzle About Infinity.” Erkenntnis, online first.

${ }^{6}$ To elaborate, here are my tentative two cents about each of these three supertasks:

The Flea. Note a crucial disanalogy with Linnebo's scale. While the scale has infinite weights and counterweights pressing on it, there are no synchronous constraints at all on the location of the flea. That means the only thing that could settle the flea's end point is the infinite winding path it takes to get there. In my view, this makes it unsurprising that the flea's end point depends on its path, and so on the order of its jumps. So in this case, I find Linnebo's prediction quite plausible, viz. that the flea ends up at $\ln (2) \approx 0.69 \mathrm{inch}$. Any alternative would involve teleportation, a violation of the cherished locality principle. This makes it reasonable to maintain the continuity of locomotion where possible, even if the more general Continuity Principle is abandoned.

Annexia. The final area of Annexia is a function of the particular regions that end up under Annexian control. Within the given parameters, Annexia could end up with $0.69 \mathrm{mi}^{2}$ of additional territory: this happens if the trades involve an ever-shrinking border region between the two nations, so that almost every location has a final claimant. But that optimal outcome is not at all guaranteed. If the swaps are more disparate, there could be large areas that are traded back and forth indefinitely, without a final claimant. Suppose ownership of those areas remains unsettled. Then, far from gaining any land, Annexia could be left without a settled claim to any territory at all, provided its neighbour is strategic enough to organise the swaps in a suitable way.

The Rod. The positive charge of the rod is generated by the electron deficit in the rod (or, if you prefer, by the proton surplus). After the first step, the rod has an electron deficit of 1 coulomb. That deficit is subsequently compensated in part by electrons added later in the process. But how many of those compensating electrons last until the end? Assuming it makes sense to re-identify token electrons (cf. Huggett 1999), the answer depends on which individual electrons are removed at each step. For suppose the electrons exit the rod in the same order as they entered. Then each electron that enters the rod during this supertask will be removed at some later step. Consequently, the charge in the rod will then be 1 coulomb or greater at the end of the task, since nothing remains to compensate the initial deficit. Alternatively, it could be that some number of newly added electrons do stick around, which could lead to any intermediate outcome between 0.69 and 1 coulomb. 